\title{
Acceptance of COVID-19 vaccination and its determinants among Lebanese dentists: a cross-sectional study
}

\author{
Lara Nasr ${ }^{1 *}$, Nadine Saleh${ }^{1}$, Mira Hleyhel ${ }^{1}$, Abbass El-Outa $^{2}$ and Ziad Noujeim ${ }^{3}$
}

\begin{abstract}
Background: Dentists are at high risk of exposure to occupational Coronavirus Disease 2019 (COVID-19). Since vaccination is crucial to control COVID-19 pandemic, we aimed to assess COVID-19 vaccination acceptance and its determinants among Lebanese practicing dentists.

Methods: A cross-sectional online study was conducted between February 15 and 22, 2021, among dentists practicing in Lebanon. Prevalence of COVID-19 vaccine acceptance was estimated. A multivariable modified Poisson regression model was used to explore determinants of COVID-19 vaccine acceptance.

Results: In total, $86 \%$ of participants were willing to receive or have already received a COVID-19 vaccine. Having received the influenza vaccine during the COVID-19 pandemic was linked to a 12\% increase in the COVID-19 vaccination acceptance rate. In addition, participants having moderate and high COVID-19 vaccination knowledge levels were more likely to accept receiving the vaccine, and participants whose fear of COVID-19 level was high were more likely to accept receiving the vaccine compared to those having a low fear level. Contrarily, those who visit the medical doctor only when needed and once a year were less likely to accept COVID-19 vaccine compared to participants who routinely visit the medical doctor.

Conclusions: Our study showed a high level of acceptance of COVID-19 vaccination among Lebanese practicing dentists. And since knowledge about COVID-19 vaccination was associated with the vaccine acceptance, it should be improved and updated to further increase the acceptance rate. High acceptability of COVID-19 vaccination among dentists is expected to have a positive impact among the population in terms of increasing awareness and vaccine uptake.
\end{abstract}

Keywords: COVID-19 vaccines, SARS-CoV-2, Vaccination, Dentists

\section{Background}

An outbreak of pneumonia of unknown cause in Wuhan, China, was first reported in December 2019, and has raised intense concerns at an international level [1]. A novel coronavirus, severe acute respiratory coronavirus 2 (SARS-CoV-2), had been identified and isolated from

\footnotetext{
*Correspondence: lar.n.92@gmail.com

${ }^{1}$ Department of Epidemiology and Biostatistics, Faculty of Public Health, Lebanese University, Fanar, Lebanon

Full list of author information is available at the end of the article
}

patients in Wuhan by January 7, 2020, and has rapidly spread from Wuhan to the rest of the world [1]. On January 30, 2020, the Coronavirus Disease 2019 (COVID-19) outbreak has been declared as a "Public Health Emergency of International Concern" by the World Health Organization (WHO) [2]. On March 11, 2020, COVID19 outbreak has been announced by the WHO as a global pandemic [2]. And up to March 25, 2021, there have been more than $123,000,000$ confirmed cases with more than 2,710,000 deaths reported to the WHO worldwide. So far, 
no treatment for COVID-19 has been curative and universally approved, and in this regard, prevention remains the only crucial mean to fight against this virus which is constantly mutating into new variants, and among preventive measures, vaccination prevails [3].

At the time of this study, several COVID-19 vaccines have already been developed using different technologies, yet only mRNA vaccines (Moderna and Pfizer/BioNTech) with high efficacy (94-95\%) and Janssen vaccine of Johnson and Johnson (one dose viral vector vaccine) have been authorized by the U.S. Food and Drug Administration (FDA) for emergency use [3-5]. COVID-19 vaccines produced by conventional methods (viral vectors, subunit particles, attenuated and inactivated viruses) have comparatively shown satisfactory but lower levels of efficacy ranging from 70 to $92 \%$ [3]. Currently, many countries have already started vaccination campaigns and Lebanon received its first batch of Pfizer/BioNTech COVID-19 vaccines on February 14, 2021; the vaccination campaign immediately started with elderly citizens and healthcare professionals (HCPs) including Lebanese dentists registered in the Lebanese Dental Association (LDA).

Oral healthcare professionals are at high risk of exposure to SARS-CoV-2 which spreads via respiratory droplets and aerosols resulting from oral surgical and dental procedures; cross-infection is the other (indirect) mode of transmission of this virus in the dental surgery [6-9]. Several measures and guidelines were adapted to clinical dental practice since the beginning of the outbreak to prevent transmissions among patients through crossinfection and transmission to and from the dentist himself/herself [7-9]. Nevertheless, the definitive solution to reduce the discussed risk, prevent further spread of the virus, and avoid complications associated with this disease, is to reach herd immunity, optimally through vaccination. Therefore, dental practitioners and their assistants are in utmost need to get vaccinated [10].

In reality, vaccines' effectiveness depends on the race to vaccinate the world's population before new resistant variants emerge, and this mainly relies on the acceptance of COVID-19 vaccination [3]. When a new vaccine is introduced, hesitancy usually arises against its safety and effectiveness. Since they are responsible to promote best health practices, HCPs' influence on the public is crucial in spreading vaccination awareness [11-13]. Therefore, it is important to evaluate their COVID-19 vaccination acceptance and knowledge. Several researchers have evaluated such parameters among healthcare workers in few countries and showed an acceptance rate ranging from 27.7 to $85 \%$ among them [14-19], but, to our knowledge, no similar study was conducted in Lebanon yet. The current economic crisis in Lebanon has rapidly worsened due to the COVID-19 pandemic's multiple lockdowns that hit hard the economy. At the time of this study, Lebanon was still far from acquiring herd immunity, which further threatened its recession, standard of living, and collapsing healthcare system, this issue being due to the unavailability of vaccines to the public yet and to the vaccine's hesitancy in many social and occupational categories of Lebanese population. In addition, healthcare professionals' COVID-19 vaccination acceptance is crucial in order to positively influence its general public acceptance and, consequently, reach herd immunity as soon as possible. And since vaccine's acceptance is context-specific and varies with many factors, we aimed to assess the prevalence of COVID-19 vaccine acceptance among Lebanese dentists practicing in Lebanon, and to evaluate their knowledge towards COVID-19 vaccination. We also aimed to determine and analyze the predicting factors for the acceptance as many characteristics were collected from participants, among them, having comorbidities, frequency of medical visits, and the fact of living with medically vulnerable individuals which may seem to influence the vaccine acceptance.

\section{Methods \\ Study design}

This observational cross-sectional online survey was conducted in Lebanon between February 15 and February 22, 2021, in order to evaluate the acceptance of the COVID19 vaccination among practicing dentists in Lebanon.

\section{Ethical considerations}

Being observational and respecting participants' anonymity and confidentiality, the Institutional Review Board (IRB) of the Lebanese University in Beirut-Lebanon waived the need for an ethical approval. Data were collected anonymously with no identifying or sensitive information. The allocated time for the questionnaire completion was around seven minutes.

\section{Study population and sample size calculation}

Participants were eligible if they were Lebanese dentists practicing dentistry in Lebanon on a full or part-time basis and registered in the Lebanese Dental Association (LDA-Beirut or LDA-Tripoli).

Using the Epi Info $^{\text {TM }}$ software, and considering that around 5000 Lebanese dentists are currently practicing dentistry in Lebanon, the minimal sample size calculated was 357 with an expected acceptance prevalence of 50\%, a confidence level of $95 \%$, and an acceptable margin of error of $5 \%$. 


\section{Data collection}

After reviewing literature [3, 10-13] and available information on COVID-19 vaccination from international guidelines $[4,5]$, a study instrument was developed in English and presented using the LimeSurvey ${ }^{\circledR}$ platform, an advanced online survey system. Expert opinions on the importance, intelligibility and clarity of the questionnaire's content were considered before the final version was distributed. The questionnaire comprised four sections:

1. Socio-demographic and work practice characteristics including age, sex, marital status, living with vulnerable individuals, main area of practice, specialty(ies), years of experience, and professional affiliation(s), ...

2. Medical history and behavior section including smoking status, frequency of medical visits, influenza and hepatitis $B$ vaccinations, chronic medical conditions, allergies, experience with COVID-19 virus, fear related to getting infected with COVID-19 virus, and general attitude towards taking medications and the concept of vaccination. Participants were asked to indicate on a visual analogue scale from 0 to 10 how much they are scared of getting infected or reinfected with COVID-19 virus.

3. COVID-19 vaccine acceptance section including questions related to having already received COVID19 vaccine (yes or no), willing to get vaccinated (yes, no, or undecided), and motivators and barriers to receiving the vaccine. COVID-19 vaccine acceptance is the main outcome dependent variable of this study that included the actual acceptance (having received the COVID-19 vaccine) and the willingness to get vaccinated if the participant had not yet received the vaccine.

4. COVID-19 vaccine knowledge questionnaire consisting of 13 true/false/don't know questions aiming to evaluate the general knowledge of dentists on COVID-19 vaccines. This questionnaire included items on misconceptions on the potential vaccination risks.

\section{Procedure}

Due to limitations in conducting face-to-face research during the current COVID-19 pandemic, the developed self-administered questionnaire was distributed online to Lebanese dentists. Invitations to participation were sent by the investigators via social networking platforms such as WhatsApp, Facebook, and Instagram using a snowball-sampling technique where participants were encouraged to pass on the questionnaire link to their colleagues. A short invitation text accompanied the survey link. Upon receiving and clicking the link, participants got automatically directed to the study aim and informed consent page, followed by the questionnaire to be filled.

\section{Statistical analysis}

All data analyses were carried out using IBM SPSS Statistics for Windows (Version 26) (IBM Corp., Armonk, NY, USA). All tests were two-tailed and a $p$-value of less than 0.05 was considered statistically significant.

To describe the sample characteristics and to summarize variables, descriptive statistics were presented as frequency/percentage and mean \pm standard deviation for categorical and continuous variables respectively. The main outcome of the study was the acceptance of COVID-19 vaccination.

For the knowledge index, a correct answer was given one (1) point and a wrong or "I don't know" answer was given zero (0) point. The total knowledge index was calculated by summing all the answers and it ranged from 0 to 13 , with a higher score indicating a better knowledge (Cronbach's alpha: 0.662). A modified Bloom's cut off point [20] was used to categorize the index into three levels of knowledge: high (80-100\%; score range: $11-13)$, moderate (50-79\%; score range: 7-10), and low (less than $50 \%$; score range: $0-6$ ). The fear of COVID-19 index was also categorized into three levels as per the previously mentioned modified Bloom's cut off point.

To explore determinants of participants' acceptance of COVID-19 vaccination, bivariate analyses were first carried out between the dependent variable (acceptance) and the independent variables (chi-square test and Student t-test for categorical and continuous variables respectively), and those having a $p$-value $\leq 0.2$ were then included in a multivariable modified Poisson regression model where adjusted prevalence ratio (aPR) values and their $95 \%$ confidence intervals (95\% CI) were calculated. The model included originally the following variables: age, years of experience in dental practice, influenza vaccination during COVID-19 pandemic, frequency of medical visits, fear levels of getting infected or re-infected with COVID-19 virus, having chronic medical condition(s), COVID-19 vaccination knowledge levels, ever had COVID-19, and living with vulnerable individuals.

\section{Results}

Out of 802 responses collected during the survey period, 253 were excluded due to incomplete data and 20 were also excluded for not meeting the inclusion criteria (being Lebanese and practicing dentistry on Lebanese territories). The remaining 529 participants were included in the study, and among them, responses regarding the knowledge section were missing in 20 
included questionnaires. Baseline characteristics of the participants are shown in Table 1. The mean age of the 529 dentists was $40.54 \pm 14.01$ years, ranging from 22 to 78 years. There were $57.8 \%$ general practitioners and $42.2 \%$ specialists; participants were given the option to choose more than one specialty including Oral and/or Maxillofacial Surgery (9.6\%), Orthodontics and Dentofacial Orthopedics (4.9\%), Restorative and Esthetic dentistry (4.0\%), Prosthodontics (6.2\%), Endodontics (5.5\%), Pediatric dentistry (4.2\%), Oral Medicine (2.1\%), Oral Pathology (2.1\%), Oral and Maxillofacial Radiology (0.9\%), and Implant dentistry (12.9\%). Participant dentists had a mean experience of $16.1 \pm 12.65$ years, ranging from 0 to 50 years.

When participants were asked if they have ever received hepatitis B vaccine, 470 (88.8\%) answered positively, among them only $20.2 \%$ were regularly checking their Hepatitis B surface antibody (anti-HBs) titers. Eighty (15.1\%) participants had at least one medical comorbidity including arterial hypertension (56.3\%), diabetes $(30 \%)$, cardiac $(22.5 \%)$, respiratory $(11.3 \%)$, autoimmune (6.3\%), thromboembolic (2.5\%), liver (2.5\%), kidney (1.3\%) diseases, and/or cancer (5\%).

Among participants who had COVID-19 (21.2\%), $60(53.6 \%)$ had mild, $44(39.4 \%)$ moderate, and 8 (7.1\%) severe symptoms. Only $26.8 \%$ out of 112 who had COVID-19 were aware of having acquired a significant level of antibodies; and $42 \%$ were still suffering from at least one symptom after recovery including fatigue $(22.3 \%)$, shortness of breath $(11.6 \%)$, olfaction disturbances (10.7\%), cough (8\%), mood swings (7.1\%), muscle pain $(7.1 \%)$, loss of gustation and/or headache (6.3\%), depressive symptoms (5.4\%), heart palpitations (4.5\%), joint pain $(3.6 \%)$, and/or chest pain (2.7\%). Participant dentists reported a mean scale of fear from getting infected or re-infected with COVID-19 virus of $6.06 \pm 2.92$, ranging from 0 to 10 . Participants who had already received the COVID-19 vaccine $(10.8 \%)$ were asked to indicate the vaccine brand taken; $86 \%, 8.8 \%$, and 5.3\% have received Pfizer/BioNTech, Sinopharm, and Sputnik V vaccines, respectively. Participants who showed willingness to get vaccinated against COVID-19 (75.2\%) were asked to indicate the vaccine brand(s) they feel comfortable to receive with a multiple-choice option: Pfizer/BioNTech (71.9\%), Sputnik V (30.2\%), Moderna (26.1\%), AstraZeneca (21.4\%), Janssen (12.3\%), Sinopharm (12.1\%), Sinovac (11.1\%), and Novavax (2.1\%).

The COVID-19 vaccination acceptance rate among participant dentists was $86 \%$. Motivators for acceptance and barriers against it are shown in Fig. 1. Main motivators (Fig. 1a) for the vaccine acceptance were desire of the participants to protect their families from getting infected $(79.8 \%)$, to protect themselves (76.9\%), wanting the pandemic to end quickly (74.3\%), and the desire to return to their normal social life $(68.4 \%)$. Barriers (Fig. 1b) against getting vaccinated mainly included concerns about the possible long-term side effects of COVID-19 vaccines (63.5\%), the rapidity of vaccines' development (58.1\%), and the effectiveness against new virus variants (50\%). COVID-19 vaccination knowledge index ranged from 0 to 13 with a mean of $8.67 \pm 2.45$; knowledge levels and scores' distribution are shown in Table 2.

The results of the modified Poisson regression analysis for determinants of COVID-19 vaccine acceptance are shown in Table 3. Having received the influenza vaccine during the COVID-19 pandemic was linked to a $12 \%$ increase in the COVID-19 vaccination acceptance rate (aPR: 1.12; 95\% CI: $1.06-1.20, p=0.001$ ). In addition, visiting the medical doctor only when needed (aPR: 0.87; 95\% CI: 0.82-0.97, $p=0.007$ ) and once a year (aPR: 0.89; 95\% CI: $0.79-0.96, p=0.004)$ were respectively associated with $13 \%$ and $11 \%$ decrease in the COVID-19 vaccine acceptance rate. Moreover, we found that having a moderate level of COVID-19 vaccination knowledge (aPR: 1.25 ; 95\% CI: 1.09-1.44, $p=0.001$ ) and having a high level (aPR: 1.30; 95\% CI: $1.32-1.50, p<0.001$ ) were related to $25 \%$ and $30 \%$ increase in the vaccination acceptance rate; similarly, participants whose fear of COVID-19 level was high were significantly more likely to accept receiving a COVID-19 vaccine compared to those having a low fear level.

\section{Discussion}

As COVID-19-related morbidity and mortality are dramatically rising worldwide, prevention by vaccination is gaining ground among HCPs. Dentists are clinical specialists with a high risk of cross-infection in dental practice, and among preventive measures recommended in dentistry, vaccination remains the most prevailing one despite vaccine's hesitancy, an emerging public challenge caused by rumors and misinformation [3, 6, 7].

Earlier studies have tackled the general population's acceptance of COVID-19 vaccination [21-28] worldwide, and given the paucity of such studies in dental communities [18], we chose to conduct a survey on Lebanese dentists' acceptance of COVID-19 vaccination.

Our acceptance rate result (86\%) is similar to that found in a previous study [18] conducted on dental professionals (85\%), and to that found in another study [19] conducted on healthcare workers that reported dentists' acceptance rate of $82.5 \%$. Studies on healthcare workers' COVID-19 vaccine acceptance in Greece [19], France and French-speaking parts of Belgium and Canada [16], and China [15], also showed comparable acceptance rates of $78.5 \%, 76.4 \%$ and $72.4 \%$ respectively. These findings 
Table 1 General characteristics of the study population ( $N=529)$

\begin{tabular}{|c|c|c|c|}
\hline Characteristics & & Frequency $(\mathrm{N})$ & Percentage $(\%)$ \\
\hline \multicolumn{4}{|l|}{ Sociodemographic and occupational characteristics } \\
\hline \multirow[t]{5}{*}{ Age groups (years) } & $20-29$ & 178 & 33.6 \\
\hline & $30-39$ & 101 & 19.1 \\
\hline & $40-49$ & 80 & 15.1 \\
\hline & $50-59$ & 111 & 21.0 \\
\hline & $>60$ & 59 & 11.2 \\
\hline \multirow[t]{2}{*}{ Sex } & Males & 292 & 55.2 \\
\hline & Females & 237 & 44.8 \\
\hline \multirow[t]{3}{*}{ Marital status } & Single & 197 & 37.2 \\
\hline & Married & 315 & 59.5 \\
\hline & Divorced/Widowed & 17 & 3.2 \\
\hline \multirow[t]{4}{*}{ Living with $^{\mathrm{a}}$} & Children & 279 & 52.7 \\
\hline & Individuals older than 65 years & 127 & 24.0 \\
\hline & Individuals with chronic diseases & 124 & 23.4 \\
\hline & Non-applicable & 114 & 21.6 \\
\hline \multirow[t]{5}{*}{ Main area of dental practice } & Northern Lebanon & 66 & 12.5 \\
\hline & Eastern Lebanon & 28 & 5.3 \\
\hline & Southern Lebanon & 55 & 10.4 \\
\hline & Beirut & 141 & 26.7 \\
\hline & Mount Lebanon & 239 & 45.2 \\
\hline \multirow[t]{2}{*}{ Specialty } & General practitioner & 306 & 57.8 \\
\hline & Specialist & 223 & 42.2 \\
\hline \multirow[t]{5}{*}{ Years of experience in dental practice } & $0-10$ & 231 & 43.7 \\
\hline & $11-20$ & 104 & 19.7 \\
\hline & $21-30$ & 112 & 21.2 \\
\hline & $31-40$ & 74 & 14.0 \\
\hline & $>40$ & 8 & 1.5 \\
\hline \multirow[t]{4}{*}{ Affiliation } & Private dental office and/or dispensary & 416 & 78.6 \\
\hline & Hospital affiliation & 20 & 3.8 \\
\hline & Academic affiliation & 52 & 9.8 \\
\hline & University postgraduate student/resident & 41 & 7.8 \\
\hline \multirow[t]{2}{*}{ Practice outside Lebanon on a part-time basis } & Yes & 42 & 7.9 \\
\hline & No & 487 & 92.1 \\
\hline \multicolumn{4}{|l|}{ Health-related characteristics } \\
\hline \multirow[t]{2}{*}{ Health coverage (medical insurance) } & Insured & 361 & 68.2 \\
\hline & Non-insured & 168 & 31.8 \\
\hline \multirow[t]{2}{*}{ Smoking status } & Smoker & 125 & 23.6 \\
\hline & Non-smoker & 404 & 76.4 \\
\hline \multirow[t]{3}{*}{ Frequency of medical visits } & Routinely & 53 & 10.0 \\
\hline & Once a year & 140 & 26.5 \\
\hline & When needed & 336 & 63.5 \\
\hline \multirow[t]{3}{*}{ Drug consumption } & I usually take when needed & 275 & 52.0 \\
\hline & I only take when prescribed & 171 & 32.3 \\
\hline & I don't like to take medications & 83 & 15.7 \\
\hline \multirow[t]{2}{*}{ Having chronic medical condition(s) } & Yes & 80 & 15.1 \\
\hline & No & 449 & 84.9 \\
\hline \multirow[t]{2}{*}{ Influenza vaccination during COVID-19 pandemic } & Vaccinated & 157 & 29.7 \\
\hline & Non-vaccinated & 372 & 70.3 \\
\hline
\end{tabular}


Table 1 (continued)

\begin{tabular}{|c|c|c|c|}
\hline \multicolumn{2}{|l|}{ Characteristics } & \multirow{2}{*}{$\begin{array}{l}\text { Frequency (N) } \\
470\end{array}$} & \multirow{2}{*}{$\begin{array}{l}\text { Percentage (\%) } \\
88.8\end{array}$} \\
\hline Hepatitis B vaccination & Vaccinated & & \\
\hline & Non-vaccinated & 59 & 11.2 \\
\hline \multirow[t]{2}{*}{ Drug allergy } & Allergic & 42 & 7.9 \\
\hline & Non-allergic & 487 & 92.1 \\
\hline \multirow[t]{2}{*}{ Other allergies (food, seasonal, latex, ...) } & Allergic & 104 & 19.7 \\
\hline & Non-allergic & 425 & 80.3 \\
\hline \multirow[t]{3}{*}{ Vaccination concept opposition } & Yes & 38 & 7.2 \\
\hline & No & 431 & 81.5 \\
\hline & Don't know & 60 & 11.3 \\
\hline \multicolumn{4}{|l|}{ COVID-19-related characteristics } \\
\hline \multirow[t]{2}{*}{ Ever had COVID-19 } & Yes & 112 & 21.2 \\
\hline & No & 417 & 78.8 \\
\hline \multirow[t]{2}{*}{ Knowing someone hospitalized or dead from COVID-19 } & Yes & 450 & 85.1 \\
\hline & No & 79 & 14.9 \\
\hline \multirow[t]{3}{*}{ Fear levels of getting infected or re-infected with COVID-19 virus } & Low level (0-4) & 118 & 22.3 \\
\hline & Moderate level (5-7) & 223 & 42.2 \\
\hline & High level (8-10) & 188 & 35.5 \\
\hline \multirow[t]{3}{*}{ Encouraged family members to get the vaccine } & Yes & 461 & 87.1 \\
\hline & No & 35 & 6.6 \\
\hline & Non-applicable & 33 & 6.2 \\
\hline \multirow[t]{2}{*}{ Already received or willing to receive COVID-19 vaccine (acceptance) } & Yes & 455 & 86.0 \\
\hline & No/undecided & 74 & 14.0 \\
\hline
\end{tabular}

" Add up to more than $100 \%$ due to multiple choice option

are substantially higher in comparison to a study conducted on Congolese healthcare workers [14], where an acceptance rate of $27.7 \%$ was found; this low rate could be explained by the fact that the survey was conducted at a time where full scientific information on COVID-19 were not yet elucidated and vaccines' clinical trials not yet launched, and according to authors, this relatively low rate may also be explained by the harm of social networks and spread of misinformation [14]. Another study [17] showing a low acceptance rate was conducted among healthcare workers in the USA: only $36 \%$ of respondents were willing to take the vaccine as soon as it becomes available, whereas $56 \%$ were hesitant of taking it or would prefer to wait for new data regarding vaccination. This study that classified healthcare workers into four major categories showed that direct medical providers had higher vaccine acceptance (49\%) compared to administrative staff (34\%), and direct patient care providers had the lowest vaccine acceptance rate (27\%) with nearly twothirds of them $(62 \%)$ choosing to postpone their vaccination after reviewing its safety data [17].

Studies targeting the general population in different countries reported COVID-19 vaccine acceptance rates of $93.3 \%, 91.3 \%, 67 \%, 64.7 \%, 53.1 \%, 37.2 \%$ and $36.8 \%$, in Indonesia, China, United States, Saudi Arabia, Kuwait,
Hong Kong, and Jordan respectively [21-26, 28], whereas a global survey has found an acceptance rate of $71.5 \%$ [27].

Our results showed a high rate of acceptance which may be explained by several factors. Being part of the high-risk group of getting COVID-19, and the exclusive availability of Pfizer/BioNTech mRNA COVID19 vaccine in Lebanon at the time of the study (which proved to have a high efficacy and safety), are possible reasons that have positively influenced Lebanese dentists' COVID-19 vaccination acceptance. This high rate may also be justified by the number of deaths due to COVID-19 in Lebanon which exceeded 5000 at the period of the survey (among them many dentists and physicians), therefore possibly influencing the fear level which, in turn, have positively affected the acceptance rate. Other potential influencing considerations included the extreme economical and financial crises that were exacerbated by the multiple total lockdowns in Lebanon, rendering the citizens desperate to return to normal professional activities. Key motivating factors for COVID-19 vaccination acceptance among dentists and barriers against it, complied with findings obtained in previous studies [17, 21-23, 25, 28]. The overbalance of motivators on the scientifically-unproven barriers 


\section{Motivators}

I want my children to resume school as soon as possible

It may become mandatory for traveling abroad

It is recommended by health authorities (WHO, Ministry of Public Health, and physicians)

I am part of the high-risk group

I believe that the benefits of vaccination outweigh its possible side effects

I am scared of the severity and complications of COVID-19

I want to return to my normal professional activities

I want to return to my normal familial and social life

I want the pandemic to end quickly

I want to protect myself from getting infected with COVD-19

I want to protect my family from getting infected with COV D-19

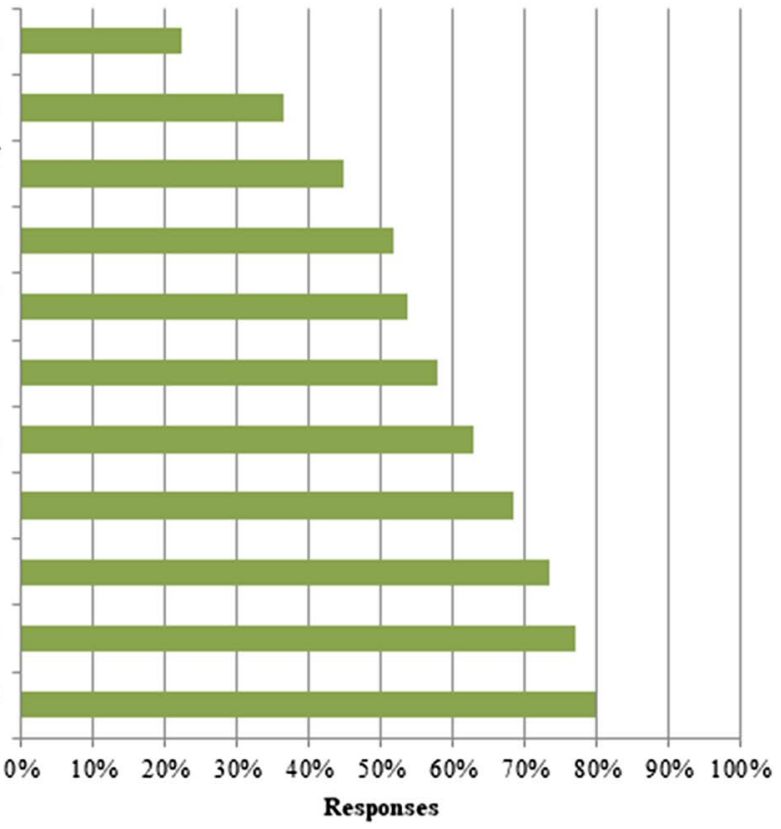

a

\section{Barriers}

I am pregnant or breastfeeding

I don't need to be vaccinated because I am young

I believe that COVID-19 vaccination is a conspiracy

I don't need to be vaccinated because I am healthy

I take all the necessary preventive measures

I already got infected with COVID-19 and this gave me enough immunity

I am concerned that mRNA vaccine may modify my DNA

I am concerned about the short-term side effects of COVID-19 vaccines

I am concerned about the COV ID-19 vaccine effectiveness

I don't trust the pharmaceutical companies that produce the vaccines

I am concerned about the ability of the Lebanese Govemment to maintain the quality of the vaccine

I am concerned about the new technologies (mRNA) used in the vaccine production

I am concerned that COVID-19 vaccine may not be effective against new strains

The time allocated for vaccines' clinical trials was too short

I am concerned about the possible long-term side effects of COV ID-19 vaccines

b

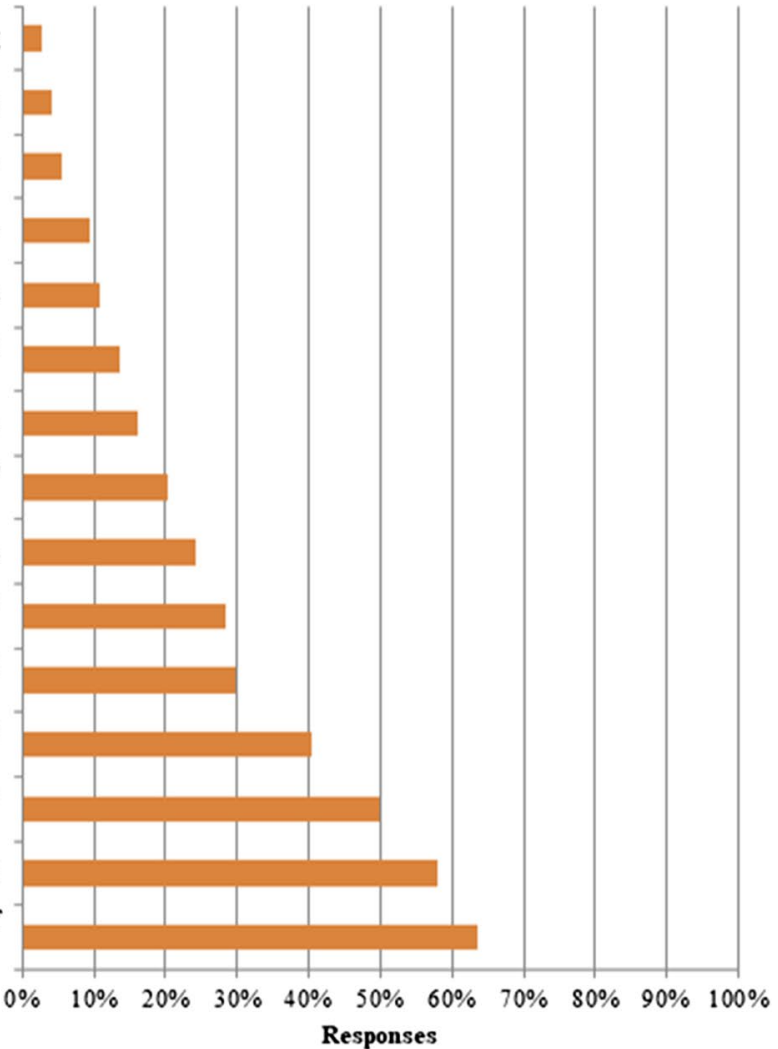

Fig. 1 a Motivators and $\mathbf{b}$ barriers for accepting COVID-19 vaccination 
Table 2 Dentists' knowledge toward COVID-19 vaccination ( $N=509)$

\begin{tabular}{|c|c|c|c|}
\hline & & Frequency $(\mathrm{N})$ & Percentage (\%) \\
\hline \multirow[t]{3}{*}{ Knowledge levels } & High (80-100\%) & 118 & 23.2 \\
\hline & Moderate (50-79\%) & 302 & 59.3 \\
\hline & Low $(<50 \%)$ & 89 & 17.5 \\
\hline Knowledge items & Answers & & \\
\hline \multirow[t]{2}{*}{ Q1-mRNA vaccine is a COVID-19 vaccine's type } & Correct & 433 & 85.1 \\
\hline & Incorrect & 76 & 14.9 \\
\hline \multirow[t]{2}{*}{ Q2-Whole virus vaccine is a COVID-19 vaccine's type } & Correct & 161 & 31.6 \\
\hline & Incorrect & 348 & 68.4 \\
\hline \multirow[t]{2}{*}{ Q3-Non-replicating viral vector is a COVID-19 vaccine's type } & Correct & 190 & 37.3 \\
\hline & Incorrect & 319 & 62.7 \\
\hline \multirow[t]{2}{*}{ Q4-Protein subunit is a COVID-19 vaccine's type } & Correct & 179 & 35.2 \\
\hline & Incorrect & 330 & 64.8 \\
\hline \multirow[t]{2}{*}{ Q5-COVID-19 vaccines decrease the risk of symptomatic infection with COVID-19 virus } & Correct & 422 & 82.9 \\
\hline & Incorrect & 87 & 17.1 \\
\hline \multirow[t]{2}{*}{ Q6-COVID-19 vaccines decrease the risk of transmission of COVID-19 virus } & Correct & 269 & 52.8 \\
\hline & Incorrect & 240 & 47.2 \\
\hline \multirow[t]{2}{*}{ Q7-Immunization is not acquired immediately after the COVID-19 vaccine injection } & Correct & 379 & 74.5 \\
\hline & Incorrect & 130 & 25.5 \\
\hline \multirow[t]{2}{*}{ Q8-All COVID-19 vaccines preparation techniques are new and were never used before } & Correct & 365 & 71.7 \\
\hline & Incorrect & 144 & 28.3 \\
\hline \multirow[t]{2}{*}{ Q9-Most of the confirmed side effects of COVID-19 vaccines are mild, resolving in 2-3 days } & Correct & 355 & 69.7 \\
\hline & Incorrect & 154 & 30.3 \\
\hline \multirow{2}{*}{$\begin{array}{l}\text { Q10-People who were previously infected and have recovered from COVID-19 will never need to } \\
\text { be vaccinated }\end{array}$} & Correct & 461 & 90.6 \\
\hline & Incorrect & 48 & 9.4 \\
\hline \multirow{2}{*}{$\begin{array}{l}\text { Q11-It is recommended to get the first and second shots of COVID-19 vaccine from two different } \\
\text { manufacturers }\end{array}$} & Correct & 423 & 83.1 \\
\hline & Incorrect & 86 & 16.9 \\
\hline \multirow[t]{2}{*}{ Q12-Preventive measures are no longer needed after vaccination against COVID-19 } & Correct & 478 & 93.9 \\
\hline & Incorrect & 31 & 6.1 \\
\hline \multirow[t]{2}{*}{ Q13-Anyone can take the COVID-19 vaccine } & Correct & 298 & 58.5 \\
\hline & Incorrect & 211 & 41.5 \\
\hline
\end{tabular}

against vaccination possibly explains the high acceptance rate obtained in our study.

Our multivariable analysis showed that the frequency of medical visits of practicing dentists is a potential predictor for COVID-19 vaccine acceptance, a finding similar to that of a Lebanese study [29] assessing regular influenza vaccination, where participants who visit their physician only when needed were found to be less likely to get vaccinated [29]. This finding could be explained by the fact that a health-conscious person would regularly visit her/his treating physician in order to remain healthy, and therefore, is more likely to get vaccinated against any life-threatening disease. Vaccination against influenza during the pandemic was found to be another positive predictor for COVID-19 vaccine acceptance, which complies with the results found in the French and Frenchspeaking parts of Belgium and Canada study [16] where participants previously vaccinated against influenza were less likely to have COVID-19 vaccine hesitancy. Our finding was also similar to that of the Chinese study [22] which reported that participants who got vaccinated for influenza in the past season had greater odds of accepting COVID-19 vaccine. The study conducted in Kuwait [25] has shown that influenza vaccination was a predictor for COVID-19 vaccine acceptance as well. At the beginning of the COVID-19 pandemic, it was thought that influenza vaccination would possibly protect against COVID19; this have possibly encouraged people who are already predisposed to accept the COVID-19 vaccination concept or any other vaccination, to receive the flu vaccine.

On the other hand, we have found in the present study that COVID-19 vaccination knowledge and fear of getting infected with SARS-CoV-2 were associated with vaccine acceptance among Lebanese dentists. Dentists who 
Table 3 Modified Poisson regression model showing predicting factors of COVID-19 vaccine acceptance

\begin{tabular}{|c|c|c|c|}
\hline Variable & $a P R^{*}$ & $95 \% \mathrm{Cl}^{* *}$ & $p$-value \\
\hline \multicolumn{4}{|c|}{ Influenza vaccination during COVID-19 pandemic } \\
\hline No (reference) & 1 & - & - \\
\hline Yes & 1.12 & $1.06-1.20$ & 0.001 \\
\hline \multicolumn{4}{|c|}{ Frequency of medical visits } \\
\hline Routinely (reference) & 1 & - & - \\
\hline Once a year & 0.89 & $0.79-0.96$ & 0.004 \\
\hline When needed & 0.87 & $0.82-0.97$ & 0.007 \\
\hline \multicolumn{4}{|c|}{ COVID-19 vaccination knowledge levels } \\
\hline Low (reference) & 1 & - & - \\
\hline Moderate & 1.25 & $1.09-1.44$ & 0.001 \\
\hline High & 1.30 & $1.32-1.50$ & $<0.001$ \\
\hline \multicolumn{4}{|c|}{ Fear levels of getting infected or re-infected with COVID-19 virus } \\
\hline Low (reference) & 1 & - & - \\
\hline Moderate & 1.07 & $0.96-1.19$ & 0.209 \\
\hline High & 1.11 & $1.002-1.227$ & 0.045 \\
\hline
\end{tabular}

Variables entered originally were: age, years of experience in dental practice, influenza vaccination during COVID-19 pandemic, frequency of medical visits, fear levels of getting infected or re-infected with COVID-19 virus, having chronic medical condition(s), COVID-19 vaccination knowledge levels, ever had COVID19 , and living with vulnerable individuals

${ }^{*}$ aPR $=$ adjusted prevalence ratio

${ }^{* *} 95 \% \mathrm{Cl}=95 \%$ confidence interval

are knowledgeable about COVID-19 vaccination such as safety, mechanism of action, ... had much less hesitancy to receive the vaccine, and this explains the positive predicting influence on the acceptance. Moreover, since vaccination is the most predictable mean to prevent infection and stop the pandemic, fearful individuals are more likely to seek vaccination in order to avoid contracting the disease.

To our knowledge, this is the first study conducted in Lebanon assessing COVID-19 vaccination acceptance among healthcare professionals and specifically, among dentists. In addition, our sample size was large enough to carry out a multivariable analysis that allowed exploring an important number of potential determinants of vaccine acceptance while controlling for confounders. Our study had some limitations. One was using the non-probability snowball sampling technique which may have generated a selection bias and may therefore have compromised the generalizability of our results. Unfortunately, it was not possible to compare participant dentists' characteristics to those of the target population due to the lack of data on the characteristics of the whole population of dentists in Lebanon. In addition, onlinesurveying method, which is restricted to computerized and internet-friendly users, had possibly created a selection bias as well, especially that those who have access to social media and internet may have better knowledge regarding COVID-19 vaccination information and increased health literacy. Furthermore, prevalence of vaccine acceptance could be overestimated because dentists not interested in getting vaccinated were possibly not motivated to participate in this survey. Also, and because our study was conducted in a period during which Lebanese dentists have started to get vaccinated, encouragements between colleagues for receiving vaccination may have had a positive impact on raising acceptability rate.

\section{Conclusions}

In the attempts to control COVID-19 pandemic, hopes are put on vaccination as the most effective preventive solution so far. Our study demonstrated a high level of acceptance of COVID-19 vaccination in Lebanese practicing dentists. Since dentists are among primary healthcare providers, such acceptance is critical and its implications are not limited to the dental professionals themselves, but may exert influence on the community and public acceptance and awareness. Together with other healthcare professionals, the duty to promote recommended public health measures against COVID-19 is a timely and difficult challenge. High acceptability of COVID-19 vaccination among dentists is expected to have a positive impact among the population in terms of increasing awareness and vaccine uptake. After all, and since knowledge about COVID-19 vaccination was found to be associated with the vaccine's acceptance, it should be improved and updated in order to further increase the acceptance rate.

Further studies are warranted to evaluate the actual vaccination rate and overall impact on dental healthcare system after the end of the vaccination campaign.

\section{Abbreviations}

aPR: Adjusted prevalence ratio; COVID-19: Coronavirus disease 2019; FDA: U.S. Food and drug administration; HCP: Healthcare professional; HCW: Healthcare worker; SARS-CoV-2: Severe acute respiratory coronavirus 2.

\section{Acknowledgements}

Not applicable.

\section{Authors' contributions}

$\mathrm{LN}, \mathrm{NS}, \mathrm{MH}, \mathrm{AEO}$, and $\mathrm{ZN}$ have equally contributed to the conception and design of the work; and to the acquisition, analysis, and interpretation of data. All authors have read and approved the manuscript.

\section{Funding}

The authors received no funding for the research reported.

\section{Availability of data and materials}

The datasets used and/or analyzed during the current study are available from the corresponding author on reasonable request. 


\section{Declarations}

\section{Ethics approval and consent to participate}

Being observational and respecting participants' anonymity and confidentiality, the Institutional Review Board (IRB) of the Lebanese University in Beirut-Lebanon waived the need for an ethical approval. As for the informed consent, participants were informed about the study purpose and they were asked to give their full consent to participate before filling the questionnaire. In addition to that, all methods were performed in accordance with the relevant guidelines and regulations ('Sex and Gender Equity in Research-SAGER—guidelines').

\section{Consent for publication}

Not applicable.

\section{Competing interests}

The authors declare that they have no competing interests.

\section{Author details}

${ }^{1}$ Department of Epidemiology and Biostatistics, Faculty of Public Health, Lebanese University, Fanar, Lebanon. ${ }^{2}$ Department of Emergency Medicine, American University of Beirut Medical Center, Beirut, Lebanon. ${ }^{3}$ Department of Oral and Maxillofacial Surgery, Faculty of Dental Medicine, Lebanese University, Hadat, Lebanon.

Received: 25 March 2021 Accepted: 16 September 2021

Published online: 29 September 2021

\section{References}

1. Wang C, Horby PW, Hayden FG, Gao GF. A novel coronavirus outbreak of global health concern. Lancet. 2020;395(Suppl 10223):470-3.

2. Cucinotta D, Vanelli M. WHO declares COVID-19 a pandemic. Acta Biomed. 2020;91(Suppl 1):157-60.

3. Fontanet A, Autran B, Lina B, Kieny MP, Karim SSA, Sridhar D. SARS-CoV-2 variants and ending the COVID-19 pandemic. Lancet. 2021:397(Supp 10278):952-4.

4. World Health Organization (WHO). COVID-19 Vaccines. [https://www. who.int/emergencies/diseases/novel-coronavirus-2019/covid-19-vacci nes] Accessed on Feb 12, 2021.

5. Centers for Disease Control and Prevention (CDC). Different COVID-19 Vaccines. [https://www.cdc.gov/coronavirus/2019-ncov/vaccines/diffe rent-vaccines.html] Accessed on Feb 12, 2021.

6. Karia R, Gupta I, Khandait H, Yadav A, Yadav A. COVID-19 and its Modes of Transmission. SN Compr Clin Med. 2020;2:1798-801.

7. Baghizadeh FM. What dentists need to know about COVID-19. Oral Oncol. 2020;105:104741

8. Checchi V, Bellini P, Bencivenni D, Consolo U. COVID-19 dentistry-related aspects: a literature overview. Int Dent J. 2021;71:21-6.

9. Banakar M, Bagheri Lankarani K, Jafarpour D, Moayedi S, Banakar MH, MohammadSadeghi A. COVID-19 transmission risk and protective protocols in dentistry: a systematic review. BMC Oral Health. 2020;20(Suppl 1):275.

10. Izda V, Jeffries MA, Sawalha AH. COVID-19: a review of therapeutic strategies and vaccine candidates. Clin Immunol. 2021;222:108634

11. Lurie N, Saville M, Hatchett $R$, Halton J. Developing COVID-19 vaccines at pandemic speed. N Engl J Med. 2020;382(Suppl 21):1969-73.

12. Paterson $P$, Meurice F, Stanberry LR, Glismann S, Rosenthal SL, Larson HJ. Vaccine hesitancy and healthcare providers. Vaccine. 2016;34(Suppl 52):6700-6.

13. Dror AA, Eisenbach N, Taiber S, Morozov NG, Mizrachi M, Zigron A, Srouji S, Sela E. Vaccine hesitancy: the next challenge in the fight against COVID-19. Eur J Epidemiol. 2020;35(Suppl 8):775-9.
14. Kabamba Nzaji M, Kabamba Ngombe L, Ngoie Mwamba G, Banza Ndala DB, Mbidi Miema J, Luhata Lungoyo C, Lora Mwimba B, Cikomola Mwana Bene A, Mukamba Musenga E. Acceptability of vaccination against COVID-19 among healthcare workers in the Democratic Republic of the Congo. Pragmat Obs Res. 2020:11:103-9.

15. Fu C, Wei Z, Pei S, Li S, Sun X, Liu P. Acceptance and preference for COVID19 vaccination in health-care workers (HCWs) (2020). https://doi.org/10. 1101/2020.04.09.20060103

16. Verger P, Scronias D, Dauby N, Adedzi KA, Gobert C, Bergeat M, Gagneur A, Dubé E. Attitudes of healthcare workers towards COVID-19 vaccination: a survey in France and French-speaking parts of Belgium and Canada, 2020. Euro Surveill. 2021;26(Suppl 3):2002047.

17. Shekhar R, Sheikh AB, Upadhyay S, Singh M, Kottewar S, Mir H, Barrett E, Pal S. COVID-19 vaccine acceptance among health care workers in the United States. Vaccines (Basel). 2021;9(Suppl 2):119.

18. Zigron A, Dror AA, Morozov NG, Shani T, Haj Khalil T, Eisenbach N, Rayan D, Daoud A, Kablan F, Marei H, Sela E, Srouji S. COVID-19 vaccine acceptance among dental professionals based on employment status during the pandemic. Front Med (Lausanne). 2021;8:618403.

19. Papagiannis D, Rachiotis G, Malli F, Papathanasiou IV, Kotsiou O, Fradelos EC, Giannakopoulos K, Gourgoulianis KI. Acceptability of COVID-19 vaccination among greek health professionals. Vaccines (Basel). 2021;9(Suppl 3):200.

20. Seid MA, Hussen MS. Knowledge and attitude towards antimicrobial resistance among final year undergraduate paramedical students at University of Gondar, Ethiopia. BMC Infect Dis. 2018;18(Suppl 1):312.

21. Harapan H, Wagner AL, Yufika A, Winardi W, Anwar S, Gan AK, Setiawan AM, Rajamoorthy Y, Sofyan H, Mudatsir M. Acceptance of a COVID-19 vaccine in Southeast Asia: a cross-sectional study in Indonesia. Front Public Health. 2020;8:381.

22. Wang J, Jing $R$, Lai $X$, Zhang $H$, Lyu Y, Knoll MD, Fang $H$. Acceptance of COVID-19 vaccination during the COVID-19 Pandemic in China. Vaccines (Basel). 2020;8(Suppl 3):482.

23. Malik AA, McFadden SM, Elharake J, Omer SB. Determinants of COVID-19 vaccine acceptance in the US. EClinicalMedicine. 2020;26:100495.

24. Al-Mohaithef M, Padhi BK. Determinants of COVID-19 vaccine acceptance in Saudi Arabia: a web-based national survey. J Multidiscip Healthc. 2020:13:1657-63.

25. Alqudeimat $Y$, Alenezi D, AlHajri B, Alfouzan H, Almokhaizeem Z, Altamim S, Almansouri W, Alzalzalah S, Ziyab A. Acceptance of a COVID-19 vaccine and its related determinants among the general adult population in Kuwait. Med Princ Pract. 2021:10:2052-61.

26. Wong MCS, Wong ELY, Huang J, Cheung AWL, Law K, Chong MKC, Ng RWY, Lai CKC, Boon SS, Lau JTF, Chen Z, Chan PKS. Acceptance of the COVID-19 vaccine based on the health belief model: a population-based survey in Hong Kong. Vaccine. 2021;39(Suppl 7):1148-56.

27. Lazarus JV, Ratzan SC, Palayew A, Gostin LO, Larson HJ, Rabin K, Kimball S, El-Mohandes A. A global survey of potential acceptance of a COVID-19 vaccine. Nat Med. 2021:27(Suppl 2):225-8.

28. Al-Qerem WA, Jarab AS. COVID-19 vaccination acceptance and its associated factors among a Middle Eastern population. Front Public Health. 2021;9:632914

29. El Khoury G, Salameh P. Influenza vaccination: a cross-sectional survey of knowledge, attitude and practices among the lebanese adult population. Int J Environ Res Public Health. 2015:12(Suppl 12):15486-97.

\section{Publisher's Note}

Springer Nature remains neutral with regard to jurisdictional claims in published maps and institutional affiliations. 